\title{
Breakthroughs in the treatment of advanced squamous-cell NSCLC: not the neglected sibling anymore?
}

\author{
Georgios Tsironis, Dimitrios C. Ziogas, Anastasios Kyriazoglou, Marita Lykka, Konstantinos \\ Koutsoukos, Aristotelis Bamias, Meletios-Athanasios Dimopoulos
}

Department of Clinical Therapeutics, Alexandra General Hospital, National and Kapodistrian University of Athens, School of Medicine, Athens, Greece

Contributions: (I) Conception and design: G Tsironis; (II) Administrative support: A Bamias, MA Dimopoulos; (III) Provision of study materials or patients: G Tsironis, DC Ziogas, A Kyriazoglou, M Lykka, K Koutsoukos; (IV) Collection and assembly of data: G Tsironis, DC Ziogas, A Kyriazoglou, M Lykka, K Koutsoukos; (V) Data analysis and interpretation: G Tsironis; (VI) Manuscript writing: All authors; (VII) Final approval of manuscript: All authors.

Correspondence to: Georgios Tsironis, MD, MSc. Department of Clinical Therapeutics, National and Kapodistrian University of Athens, School of Medicine, 80 Vas. Sofias Avenue, Athens 11528, Greece. Email: g_tsironis@hotmail.com.

\begin{abstract}
During the last years, translational research has contributed in many advances in the treatment of non-small cell lung cancer (NSCLC) discovering genetic alternations or recognizing the immunoescape and neo-angiogenesis of lung cancer. Although the majority of these advances took place in the nonsquamous histological subtype, therapeutic options for patients diagnosed with advanced squamous cell lung cancer (SqCLC) have been also enriched significantly with the addition of nab-paclitaxel in the conventional chemotherapy; the introduction of necitumumab, afatinib and erlotinib in the inhibition of epidermal growth factor receptor (EGFR) axis and of ramucirumab in the inhibition of VEGF-induced angiogenesis and last with the approvals of nivolumab, pembrolizumab atezolizumab and durvalumab soon in the promising field of immunotherapies. Agents targeted various other pathways including FGFR, IGF-1, PI3K, CDK4/6, MET and PARP inhibitors are under investigation in order to open new prospects in the treatment of SqCLC. In this review, we present all published data that led to recent approvals for the treatment of advanced SqCLC and all ongoing clinical trials that keep searching for new molecular targets following a more-personalized approach.
\end{abstract}

Keywords: Non-small cell lung cancer (NSCLC); squamous cell lung cancer (SqCLC); targeted therapy; immunotherapy

Submitted Dec 20, 2017. Accepted for publication Jan 19, 2018.

doi: $10.21037 /$ atm.2018.02.18

View this article at: http://dx.doi.org/10.21037/atm.2018.02.18

\section{Introduction}

Lung cancer remains the most common cancer diagnosis and the leading cause of cancer-related deaths, with approximately 1.8 million new cases and 1.59 million deaths accounting every year worldwide (1). During 2017, only in the USA the newly diagnosed cases and the estimated deaths were 222,500 and 155,870 respectively (2). Nonsmall cell lung cancer (NSCLC) covers the $80 \%$ of these lung cancer cases and mainly includes two large histological subsets, adenocarcinoma (46.6\%) and squamous cell lung cancer (SqCLC) (23.2\%) (3). Clinicopathologically, SqCLC differs remarkably from adenocarcinoma since its lesions are usually located more centrally, arose segmentally involving lobar or main bronchi and often showing central cavitation. The distinct histological nature of SqCLC and its different molecular background determine the variable therapeutic behavior of the disease to novel agents. Targeting genomic alterations, such as epidermal growth factor receptor (EGFR) mutations (4-8), anaplastic lymphoma kinase (ALK) 
gene rearrangements (9-12) and more recently ROS1 (13), and $B R A F$ (14) led to the approval of 9 novel agents and radically changed the treatment of patients with NSCLC from conventional chemotherapy to a more individualized molecularly-targeted approach. However, these gene alterations are rarely detected in patients with SqCLC and these patients are less benefited by the targeted therapies compared to patients with lung adenocarcinoma. Thus, the conventional platinum-based chemotherapy remains up to recently the main $1^{\text {st }}$-line option for patients with SqCLC, but the introduction of immune checkpoint inhibitors enriched the therapeutic armamentarium of medical oncologists and signaled the beginning of a new era in the management of lung cancer.

In this review, we will focus on the latest advances in the treatment of metastatic/advanced squamous-cell NSCLC and present all approved anti-VEGF and anti-EGFR agents as well as all recently developed immunotherapies that changed the landscape of SqCLC treatment during the last years.

\section{Chemotherapy}

The combination of cisplatin or carboplatin with a third generation cytotoxic agent such as paclitaxel, docetaxel, vinorelbine, or gemcitabine consists the backbone of frontline approach for advanced SqCLC according to ESMO guidelines (15). No clinical trials or meta-analyses recognized any superior combination among them $(16,17)$. Comparing the two most commonly used doublets, cisplatin-gemcitabine with cisplatin-pemetrexed a phase III non-inferiority study enrolled chemotherapy-naive NSCLC patients and stratified them according to the histological type. This study reached to prolonged survival times of cisplatin-gemcitabine arm (median progressionfree survival, PFS 5.5 vs. 4.4 months, and median overall survival, OS 10.8 vs. 9.4 months, $\mathrm{P}=0.05$ ) in the SqCLC population; whereas in the subgroup of patients with nonsquamous NSCLC, the regimen of cisplatin-pemetrexed was found more tolerable and effective (median PFS 5.3 vs. 4.7 months, and median OS 11.8 vs. 10.4 months, $\mathrm{P}=0.03$ ). Based on these outcomes, the doublet of cisplatingemcitabine received a clear indication for $1^{\text {st }}$-line treatment of SqCLC (18).

An additional approved chemotherapeutic agent for the treatment of advanced/metastatic SqCLC is nabpaclitaxel (an albumin bound form of paclitaxel). In $1^{\text {st }}$ line setting, the doublet of carboplatin with nab-paclitaxel was compared by a phase III study with standard (solventbased) paclitaxel plus carboplatin in 1,052 patients with advanced NSCLC (19). The regimen of carboplatin with nab-paclitaxel was found more effective achieving an overall response rate $(\mathrm{ORR})$ of $41 \%$ vs. $24 \%(\mathrm{P}<0.001)$ but not reaching to statistically significance for PFS and OS. The doublet of carboplatin and nab-paclitaxel presented lesser grade 3/4 toxicities compared to the conventional combination of carboplatin plus paclitaxel including mainly peripheral neuropathy, (3\% vs. 12\%) and musculoskeletal pain $(13 \%$ vs. $25 \%)$. The same combination of carboplatin and nab-paclitaxel has been compared with the doublet of gemcitabine plus carboplatin in a phase II trial of 120 untreated patients with advanced SqCLC patients and the results are still expecting (20). The role of this nanoparticle albumin-bound taxane in second line is also under investigation by an ongoing phase III study (21).

According to studies conducted mainly in Japanese population, two additional agents, the oral fluoropyrimidine derivative S-1 and the second-generation platinum analog nedaplatin, showed efficacy in SqCLC but have not included yet into the ESMO or NCCN guidelines. A combination of the first agent with carboplatin was compared with carboplatin/paclitaxel in frontline approach by a noninferiority trial resulting in a median OS of 15.2 and 13.3 months in the experimental and control arm respectively $(\mathrm{P}=0.002)$ (22). Particularly for squamous histology, median OS was 14.0 and 10.6 months in the carboplatin/S-1 and carboplatin/paclitaxel arm respectively $(\mathrm{P}=0.093)$ (23). The $\mathrm{S}-1$ derivative was also examined in combination with cisplatin versus cisplatin-docetaxel in a mixed histology NSCLC population including similar proportions of patients with SqCLC in the experimental and control arm respectively (16.6\% and $16.3 \%)$. The regimen containing S-1 was found non-inferior to the regimen of docetaxel regarding OS (median, 16.1 vs. 17.1 months) and PFS (median, 4.9 vs. 5.2 months), but showed better tolerability (significantly lower febrile neutropenia-1.0\% vs. $7.4 \%, \mathrm{P}<0.001$; grade $3 / 4$ neutropenia $22.9 \%$ vs. $73.4 \%, \mathrm{P}<0.001$; grade $3 / 4$ infection $5.3 \%$ vs. $14.5 \%$; grade $1 / 2$ alopecia $12.3 \%$ vs. $59.3 \%, \mathrm{P}<0.001)$ and improved quality of life (global health status/QoL functioning, $\mathrm{P}<0.0001)$ (24). In parallel, the second-generation platinum analog nedaplatin was compared with cisplatin, plus docetaxel, in Japanese cases with advanced or relapsed SqCLC presented a favorable safety profile (grade $\geq 3$ toxicities: nausea $4.0 \%$ vs. $14.3 \%$, fatigue $3.4 \%$ vs. $10.9 \%$, hyponatremia $13.6 \%$ vs. $30.3 \%$, hypokalemia $2.3 \%$ vs. 
8.6\%) and a higher efficacy as depicted with a significant prolongation of median OS for the experimental arm (13.6 vs. 11.4 months; $\mathrm{P}=0.037$ ) (25). Nedaplatin was also examined in advanced SqCLC by a phase II trial, as a doublet with irinotecan, but the preliminary results did not justify further investigation (26). Finishing the reporting of available chemotherapeutic options, cabazitaxel is tested in a mixed NSCLC population (containing $28.3 \%$ SqCLC patients) by a phase II study in $2^{\text {nd }}$ and $3^{\text {rd }}$ line setting (progression after docetaxel) and found well-tolerable (95\% treatment compliance, $13 \%$ dose reductions), achieving an ORR of $50 \%$, a median PFS of 2.1 months and a median OS of 7.4 months (27).

\section{Targeted therapies}

\section{Anti-VEGF treatment}

Based on the results of a phase III trial, bevacizumab has been approved for NSCLC in combination with paclitaxel/carboplatin, offering a median OS benefit of 2 months (28). However, this pivotal trial had not included patients with SqCLC due to safety concerns about lifethreatening pulmonary hemorrhage, observed in the earlier phase II trial, mainly at centrally-located SqCLC lesions (29). Radiographically, tumor cavitation was the only finding, identified as a potential risk factor for hemorrhagic events (30). Given these findings, bevacizumab is currently contraindicated in tumors of squamous histology. The risk of bleeding did not stop the further investigation of antiangiogenic effect in SqCLC. In the randomized phase III REVEL study another compound of anti-VEGF treatment, the IgG1 monoclonal antibody (mAb) of the extracellular domain of VEGFR-2, called ramucirumab was compared with placebo, as a $2^{\text {nd }}$ line doublet with docetaxel in both squamous and non-squamous stage IV NSCLC patients. The regimen of taxane plus ramucirumab was found significantly superior to taxane-placebo combination both for PFS (median, 4.5 vs. 3.0 months, $\mathrm{P}<0.0001$ ) and $\mathrm{OS}$ (median, 10.5 vs. 9.1 months, $\mathrm{P}=0.023$ ). This $\mathrm{OS}$ benefit was also retained for the SqCLC subgroup (median, 9.5 vs. 8.2 months) (31). These results led to the approval of ramucirumab for both histologies.

Keep targeting VEGF pathway, axitinib, a novel panVEGFR inhibitor (e.g., inhibitor of all three VEGFR-1, -2 and -3 ) was evaluated by a phase II trial and compared with the standard $1^{\text {st }}$-line regimen of cisplatin and gemcitabine in patients with SqCLC. Treated patients reached to a median
PFS of 6.2 months and to a median OS of 14.2 months, while the most usually presented $\geq 3$ grade toxicities were neutropenia (13.2\%) and hypertension (13.2\%). Only 3 (7.9\%) patients experienced hemoptysis, one of which fatal (2.6\%) (32).

\section{Anti-EGFR agents}

In contrast to adenocarcinoma, SqCLC is rarely characterized by EGFR mutations. Although it is not often mutated, EGFR can be overexpressed or presented with gene copy alterations in $60-80 \%$ and $7-10 \%$ of squamous lung tumors, respectively $(33,34)$. Based on these data, several anti-EGFR agents have been tested in patients with SqCLC, but unfortunately in the context of clinical trials including patients with NSCLC of all histologies. Two EGFR tyrosine kinase inhibitors (TKIs), erlotinib and afatinib and one mAb, necitumumab are the only agents targeting EGFR axis that have been approved for patients with SqCLC; the TKIs in $2^{\text {nd }}$-line and the mAb in $1^{\text {st }}$-line treatment (15). The SQUIRE study enrolled only SqCLC patients to receive the standard cisplatingemcitabine combination plus or not necitumumab as $1^{\text {st }}$ line approach. The combination of anti-EGFR mAb with chemotherapy presented longer OS (median, $11.5 \mathrm{vs.}$ 9.9 months, $\mathrm{P}=0.012$ ), longer PFS (median, 5.7 vs. 5.5 months, $\mathrm{P}=0.020$ ) and increased disease control rate ( $82 \%$ vs. $77 \% ; \mathrm{P}=0.043$ ) but additional toxicity of at least one grade 3 or worse $\mathrm{AE}(72.1 \%$ vs. $61.6 \%)$ including mainly rash and hypomagnesaemia. This unfavorable safety profile of triplet with anti-EGFR $\mathrm{mAb}$ was clinically translated with higher treatment discontinuations (31.2\% vs. $24.6 \%$ ) in this arm (35).

Erlotinib was approved after $1^{\text {st }}-$ or $2^{\text {nd }}$-line treatment of SqCLC based on the results of BR.21 trial that showed a statistically significant prolongation of OS compared to placebo in the overall population of NSCLC $(6.7 \mathrm{vs}$. 4.7 months, $\mathrm{P}<0.001$ ) and in the SqCLC subgroup (median, 5.6 vs. 3.6 months, $\mathrm{P}=0.07$ ) (36). Afatinib acts in $E F G R$ mutated-NSCLC (6) binding to the ErbB intracellular domain and inhibiting heterodimerization of the ErbB receptor. This EGFR TKI entered into ESMO Guidelines for SqCLC, through a single phase-III trial, named LuxLung 8 including only patients with squamous histology. In this study afatinib was compared with erlotinib, resulting in a statistically significant difference in PFS (2.6 vs. 1.9 months; $\mathrm{P}=0.010)$, ORR (5.5\% vs. $2.8 \% ; \mathrm{P}=0.055)$ and median OS (7.9 vs. 6.8 months; $\mathrm{P}=0.008)$ in favor of the 
Table 1 Selected trials of agents targeting EGFR axis

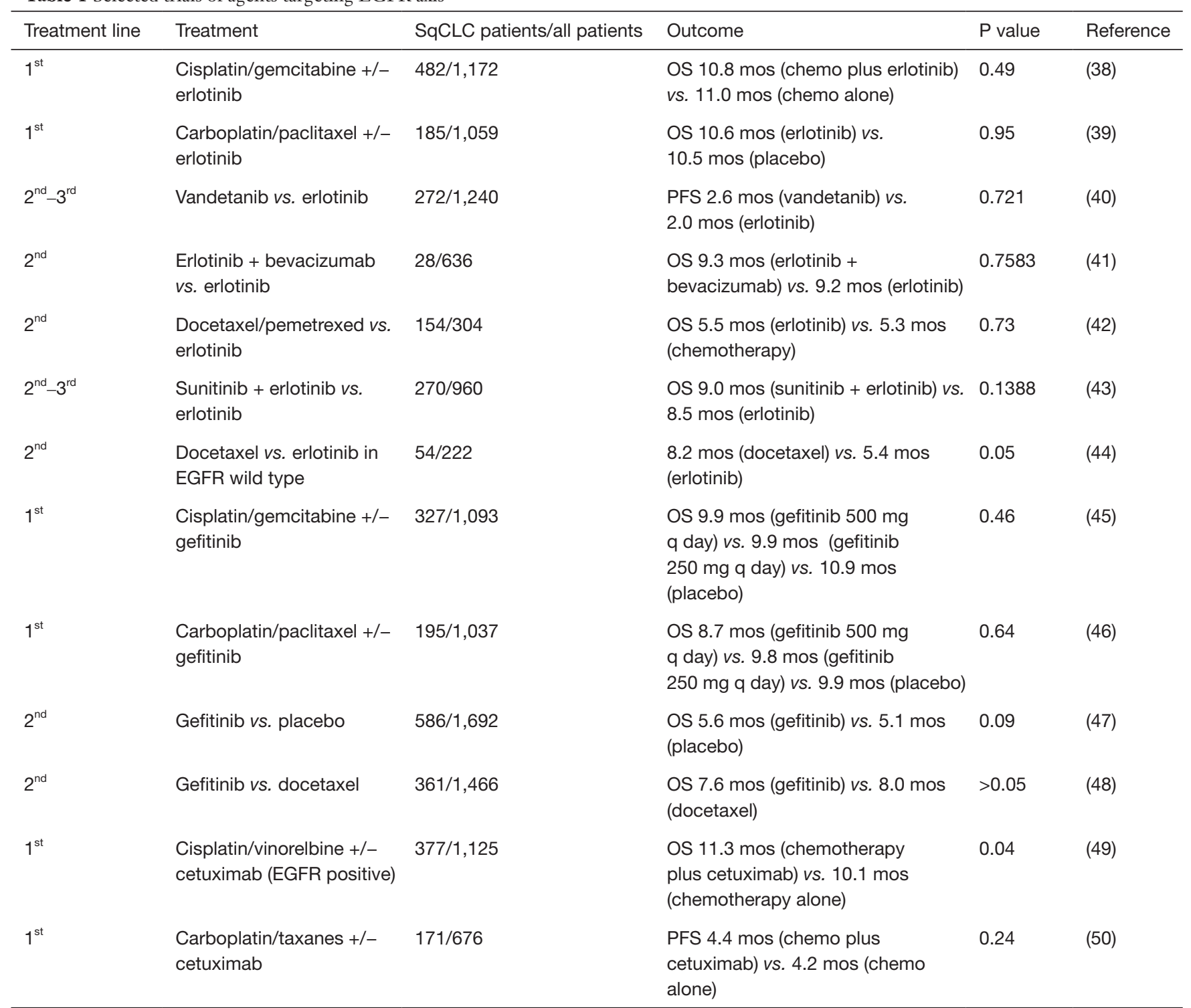

SqCLC, squamous cell lung cancer; EGFR, epidermal growth factor receptor; mos, months.

experimental arm, with no major tolerance differences, although afatinib-treated patients presented more grade $3 / 4$ diarrhea $(10 \% / 0.5 \%$ vs. $2 \% / 0.3 \%)$ and grade 3 stomatitis (4.1\% vs. 0\%) (37). Table 1 presents the most important clinical trials investigating anti-EGFR treatments (either alone or in combinations with chemotherapy or other targeted agents) in patients with mixed NSCLC histology (38-50). The small number of clinical trials including exclusively SqCLC populations and the absence of strong results could not support and upgrade the use of anti-EGFR agents as frontline therapeutic decision for patients with SqCLC. Thus, anti-EGFR agents are classified at the lower rungs of ESMO Magnitude of Clinical Benefit Scale (51).

\section{Other molecular examined pathways}

The amplification of the FGFR pathway is recognized in about $12 \%$ of SqCLC cases (52) and found to be associated with worse prognosis (53). This finding leaded to further investigation of novel FGFR targeted molecules in patients 
with NSCLC including patients with SqCLC. In this category, nintedanib is the only approved agent limited to adenocarcinoma patients as the phase III trial, LUMELung 1 that enrolled NSCLC patients to receive docetaxel $+/-$ nintedanib as $2^{\text {nd }}$-line treatment, resulted in a median OS prolongation for adenocarcinoma patients and especially those progressing in less than nine months after $1^{\text {st }}$-line (10.9 vs. 7.9 months, $\mathrm{P}=0.0073$ ) but not for the total study population (median 10.1 vs. 9.1 months, $\mathrm{P}=0.2720$ ) (54). Dovitinib is a multiple inhibitor of FGFR axis (FGFR1-3, VEGFR1-3, PDGFR $\beta$, c-KIT and FLT3), but its results from a phase II trial in pretreated patients with exclusively FGFR-amplified SqCLC, (ORR: 11.5\%, median OS 5.0 months and median PFS 2.9 months) were not so promising (55). Other agents such as AZD4547 and BGJ398 have only phase I results $(56,57)$ and NCT01761747 trial of ponatinib had been early terminated due to safety reasons (58). Therefore, no additional agents except of nintedanib targeting the FGFR axis are expecting to be approved soon for NSCLC treatment.

In SqCLC tumors (47\%) there are some alterations in the intracellular protein compounds of the PI3K-AktmTOR pathway that implicated in tumor growth (52). A phase II trial enrolled 63 NSCLC patients with PI3K activation and treated them with buparlisib monotherapy but failed to demonstrate strong efficacy (59). Another PI3K inhibitor, labeled as PX-866, was tested in combination with docetaxel in $2^{\text {nd }}$-line setting, by a phase II trial of NSCLC, containing SqCLC cases but had also negative results (60). Taselisib is the last addition in the list of investigated PI3K inhibitors as $2^{\text {nd }}$-line options but the phase II trial in patients with PI $3 \mathrm{~K}$ activated SqCLC is still ongoing (58).

Increased levels of free IGF-1 have been identified in squamous lung tumors (61). However, all investigated regimens targeting IGF-1 implicated pathway were not found significantly effective either as $1^{\text {st }}$-line treatment or as later options. For instance, figitumumab combined with paclitaxel and carboplatin in not previously treated patients with non-adenocarcinoma lung cancer had shown no superior efficacy in a phase III trial (62) while the IGF-1R modulation by AXL-1717 despite the improved frequency of severe neutropenia compared to docetaxel in a phase II trial of pretreated patients with squamous histology, had similar frequencies of non-progression at week $12(25.9 \%$ vs. 39\%) (63).

Based on the impressive results of the approved CDK4/6 inhibitors in patients with breast cancer (64), the role of cyclin dependent kinases (CDK) including CDK4 and CDK6 in the Rb1 pathway is also evaluated in the treatment of NSCLC. Specifically in SqCLC, the CDK$\mathrm{Rb} 1$ pathway is deregulated via multiple ways $(65,66)$. The first-in-class agent, palbociclib, was investigated in a singlearm phase II trial in 19 recurrent NSCLC patients, negative for p16 expression. The median PFS was 12.5 weeks and disease was stable in $50 \%$ of patients. This response was not correlated with histological type or EGFR mutational status (67).

In parallel, MET alternations found to be associated with poor prognosis through resistance to EGFR TKIs in lung adenocarcinoma and are recognized in approximately $6-10 \%$ of SqCLC, $(68,69)$. Several MET inhibitors have been evaluated in SqCLC patients with modest results to date. Onartuzumab, a mAb directed against MET has shown minimal efficacy, either in a phase II trial in combination with platinum-doublet chemotherapy as $1^{\text {st }}$-line approach of SqCLC, or in a phase III trial in combination with erlotinib in a selected pretreated population $(70,71)$. Two other molecules that inhibited MET pathway, cabozantinib and tivantinib have been tested but their combinations with erlotinib had favorable preliminary results only limited to non-squamous $K R A S$-mutated and $E G F R$ wild-type NSCLC patients, respectively $(72,73)$.

Regarding the proteasome inhibition, the outcomes of a phase II trial after the addition of bortezomib to gemcitabine and cisplatin in chemo-naive patients with SqCLC were also disappointing (ORR: 17\%, median PFS: 2.5 months and median OS: 10.6 months) (74).

Among the other non-approved but investigated agents, veliparib, an oral PARP inhibitor, showed the most promising results of phase II testing. By disabling DNA single-strand breaks repair, PARP inhibitors presented great antitumor activity in ovarian cancer especially as maintenance after response to platinum containing chemotherapy $(75,76)$. In general, SqCLC is considered a platinum-sensitive tumor, so PARP inhibitors may take a place in the sequence of its treatments. Veliparib was studied in combination with paclitaxel and carboplatin, in the first line approach of NSCLC patients (49\% of patients enrolled were SqCLC) by a placebo-controlled phase II trial. The results of this trial were more favorable for the experimental triplet compared to the standard carboplatin/ paclitaxel doublet, also in the squamous histology subgroup, presenting a median PFS of 6.1 vs. 4.1 months and a median 
OS of $10.3 v s .8 .5$ months (77).

\section{Immunotherapy}

After the fundamental paper of Hanahan and Weinberg in Cell (78), where the tumor-induced immunosuppression was recognized as an emerging hallmark of cancer, the immunotherapy was introduced as a new breakthrough anticancer treatment and exponentially administered in several tumor types and treatment lines, from melanoma (79) and lung (80) to unknown primary (81) and from metastatic to adjuvant setting $(82,83)$ changing in total the clinical approach of oncological patients. Particularly in NSCLC, many years after the initial clinical trials of immunotherapies, it is important to notice that the use of immunotherapy not only improved the quality of life but also multiplied the overall and the disease-free survival of treated patients (84).

\section{Nivolumab}

Nivolumab was the first immunotherapy agent that received approval for the second line approach of SqCLC, based on the results of CheckMate 017 that included exclusively patients with SqCLC and compared nivolumab versus docetaxel in the $2^{\text {nd }}$-line setting after a platinum doublet as $1^{\text {st }}$-line. This phase III, randomized trial resulted in a statistically significant improvement of ORR (20\% versus 9\%) and a survival benefit (median OS: 9.2 months, 95\% CI: $7.33-12.62$ vs. 6.0 months, $95 \%$ CI: $5.29-7.39$ and 2-year OS\%: $24 \%$ vs. $8 \%$ ) for the immunotherapy arm. In contrast with the non-squamous subset, PD-L1 expression was not associated with efficacy of nivolumab and notably, no survival or response advantage was found in different levels of PD-L1 (1\%, 5\% and 10\%). As expected, nivolumab presented a more tolerable safety profile compared to conventional $2^{\text {nd }}$-line chemotherapy with lesser toxicities of any grade $(59 \%$ vs. $87 \%$ ) and lesser toxicities of grade $3 / 4$ ( $8 \%$ vs. $56 \%$ ) while treatment discontinuation rates were $5 \%$ and $10 \%$, respectively $(85,86)$.

Before the pivotal CheckMate 017 trial, nivolumab has been initially evaluated in the third-line and beyond setting, in a phase II, single-arm study CheckMate 063 that enrolled 117 SqCLC patients who had progressed after a platinum-doublet and one more systematic treatment resulting in ORR of $14.5 \%$, median OS of 8.2 months, 12 - and 18 -month OS rates $39 \%$ and $27 \%$, respectively. Regarding safety, $75 \%$ of patients experienced AEs of any grade and $17 \%$ grades $3-4$ whereas immunotherapy had to be terminated due to toxicity in $17 \%$ of them (87).

Trying to improve further the depth and the duration of response, nivolumab has been tested in combinations with an anti-CTLA4 agent, ipilimumab and several other established chemotherapy doublets. However, the preliminary results of the nivolumab-ipilimumab doublet emerged some safety concerns as ipilimumab, at melanomaestablished doses of $3 \mathrm{mg} / \mathrm{kg}$ every 3 weeks in combination with nivolumab was not found well-tolerated, leading to high rates of AEs (49\%) and even to therapy-related deaths (88). Consequently, the "nivo-ipi" combination was re-evaluated at a different dose-schedule administration of ipilimumab at $1 \mathrm{mg} / \mathrm{kg}$ either every 6 or 12 weeks in 77 NSCLC patients (including 13 SqCLC patients) as $1^{\text {st }}$-line treatment with ORR of $38 \%$ and $47 \%$, respectively for the 6- and 12-week schedule. Interestingly when a PDL1 expression cut-off level of at least $1 \%$ was used ORR was found even better for both schedules (approximately 57\%). Data for 1-year OS\% were mature only for the 6-week cohort reaching to $69 \%$ (89). Immunotherapy-related AEs greater than grade 3 occurred in about 35\% in both doseschedules. This particular immuno-combination is now being evaluated in a phase II trial that has completed accrual and results are expecting to be published soon (90).

Nivolumab at $10 \mathrm{mg} / \mathrm{kg}$ has also been evaluated in combination with three different chemotherapy doublets (pemetrexed-cisplatin for adenocarcinoma, gemcitabinecisplatin for SqCLC and paclitaxel-carboplatin for all NSCLC histologies) and as monotherapy at $5 \mathrm{mg} / \mathrm{kg}$ in a multi-cohort phase I trial (91) resulting in ORR of $47 \%$, $33 \%$ and $47 \%$ for the nivo-cis-pem, nivo-cis-gem and nivo-carbo-taxol cohorts, respectively and in $43 \%$ at the half-dose of nivo monotherapy, irrespectively of PD-L1 status. The 2-year OS\% for the three immuno-chemo combinations were $33 \%$ for cis-pem, $25 \%$ for cis-gem, $27 \%$ for carbo-taxol and reached to $62 \%$ for the half-dose nivolumab. All immuno-chemo combinations presented similarly impaired safety profile. For example, $45 \%$ of treated patients reported grade $3 / 4$ toxicities and $21 \%$ had to discontinue their treatment.

\section{Pembrolizumab}

Pembrolizumab is the only checkpoint inhibitor approved in both $1^{\text {st }}$ - and $2^{\text {nd }}$-line treatment of NSCLC expressed PD-L1 in more than $50 \%$ and $1 \%$ of tumor cells (TC), respectively for the two settings. These indications based on 
the results of the Keynote 024 and 010 trials that in contrast to nivolumab studies, enrolled patients with both squamous and non-squamous lung cancer.

As frontline treatment, pembrolizumab at a fixed dose of $200 \mathrm{mg}$ was compared with a platinum-based doublet (investigator's decision, out of five different options), in 305 patients with NSCLC (56 with SqCLC histology-18\% in each arm) expressing more than 50\% PD-L1. Results were in favor of immunotherapy arm regarding median PFS (10.3 vs. 6.0 months, $\mathrm{P}<0.001)$ and 6 -month $\mathrm{OS} \%$ (80.2\% vs. $72.4 \%, \mathrm{P}=0.005$ ). In the $\mathrm{SqCLC}$ subpopulation the risk of disease progression or death was significantly reduced in immunotherapy arm (HR $=0.35,95 \%$ CI: $0.17-$ 0.71). Similar to other immunotherapies, pembrolizumab appeared fewer toxicities compared to chemotherapy arm (any grade $73.4 \%$ vs. $90.0 \%$ and grade $3 / 426.6 \%$ vs. $53.3 \%$, respectively). Most immunotherapy-related AEs were early detected at grade 1 or 2 and well managed with no recorded immunotherapy-related deaths. The superior efficacy and safety results of immunotherapy compared to standard platinum-containing doublet led to the early publication of findings and supported the approval of pembrolizumab as the recommended $1^{\text {st }}$-line regimen for patients with NSCLC expressed $>50 \%$ PD-L1 (92).

In $2^{\text {nd }}$-line setting, pembrolizumab was evaluated after progression in a platinum-containing doublet by Keynote 010 phase II/III trial. This trial enrolled 1,034 patients with NSCLC (222 SqCLC), expressing PD-L1 at least $1 \%$. Patients were randomized to receive: (I) either lowdose pembrolizumab (2 mg/kg, $\mathrm{n}=345)$, (II) high-dose pembrolizumab (10 mg/kg, $\mathrm{n}=346)$, or (III) standard dose docetaxel $\left(75 \mathrm{mg} / \mathrm{m}^{2}, \mathrm{n}=343\right)$ every 3 weeks. Immunotherapy at both doses was found more efficient and less toxic than chemotherapy. The risk of death was significantly reduced in both immunotherapy arms $(\mathrm{HR}=0.71,95 \%$ CI: $0.58-0.88, \mathrm{P}=0.0008$ and $\mathrm{HR}=0.61,95 \%$ CI: $0.49-$ $0.75, \mathrm{P}<0.0001$ for the low- and high-dose schedules, respectively) (93). Median OS was prolonged either for patients with PD-L1 expression $\geq 50 \%$ or $\geq 1 \%$ for both dosing schedules. For patients with tumors expressing PD-L1 $\geq 50 \%$, the median OS of pembrolizumab at $2 \mathrm{mg} / \mathrm{kg}$, was $14.9 \mathrm{vs}$. 8.2 months for docetaxel (HR $=0.54,95 \%$ CI: $0.38-0.77$ ) and for patients with tumors expressing PD-L1 $\geq 1 \%$, the median OS was $9.4 v s .8 .6$ months for docetaxel $(\mathrm{HR}=0.79$, 95\% CI: 0.61-1.04). The median OS for the higher doses was 17.3 vs. 8.2 months (HR $=0.50,95 \%$ CI: $0.36-0.70$ ) compared to docetaxel for the $1-49 \%$ PDL1 expression and 10.8 vs. 8.6 months (HR $=0.71,95 \%$ CI: $0.53-0.94$ ) for the $\geq 50 \%$ PDL1 expression. The PD-L1 inhibition appeared again more tolerable than chemotherapy with less grades $3 / 4$ AEs (13\% for low-dose pembrolizumab, 16\% for highdose pembrolizumab and $35 \%$ for chemotherapy) (93).

Aiming in the improvement of efficacy, trials are evaluating pembrolizumab in different combinations with either targeted therapy, or chemotherapy or other immunemodulating agents. Keynote 021 is a multi-cohort phase 1/2 randomized trial that enrolled NSCLC patients to receive one of the following combinations: carboplatin-paclitaxelpembrolizumab or paclitaxel-carboplatin-bevacizumabpembrolizumab or carboplatin-pemetrexed-pembrolizumab or ipilimumab-pembrolizumab or erlotinib-pembrolizumab or gefitinib-pembrolizumab. Results are announced and led to the approval of carboplatin-pemetrexed-pembrolizumab due to a significant PFS prolongation for patients receiving the combination but will not be further analyzed as only non-SqCLC patients were enrolled at this cohort (94).

\section{Atezolizumab}

Atezolizumab is a humanized IgG1-kappa mAb that binds PD-L1 and inhibits PD-L1/PD-1 and PD-L1/B7.1 interactions. It was subsequently approved for patients with NSCLC, in $2^{\text {nd }}$-line setting. Atezolizumab was initially evaluated in a phase II randomized trial, where 277 patients (97 cases with SqCLC) were randomized to receive atezolizumab $1,200 \mathrm{mg}$ or docetaxel every 3 weeks, resulting in a 3 -month prolongation of overall survival in favor of immunotherapy arm (12.6 vs. 9.7 months, $\mathrm{P}=0.04)$. The ORR and the duration of response for the immunotherapy arm were $15.3 \%$ and 18.6 months respectively (95). Atezolizumab was further evaluated in a phase III (OAK) study that enrolled 1,225 NSCLC patients of squamous (222 cases) and non-squamous (628 cases) histology to receive atezolizumab $1,200 \mathrm{mg}$ or docetaxel at standard dose after failure of platinum-based $1^{\text {st }}$-line chemotherapy. PD-L1 expression was evaluated not only in TC but also in tumor infiltrating immune cells (IC) in order to recognize a combinatorial marker that could predict response to immunotherapy. Median OS was significantly prolonged in the whole cohort and in the subgroups of PD-L1 expression. More specifically, for expression $\geq 1 \%$ PD-L1 (TC or IC) the OS was 15.7 vs. 10.3 months $(\mathrm{P}=0.010)$; for expression $<1 \% \mathrm{PD}-\mathrm{L} 1$ (TC or IC) 12.6 vs. 8.9 months $(\mathrm{P}=0.022)$; for high $\mathrm{PD}-\mathrm{L} 1$ ( $\mathrm{TC} \geq 50 \%$ or IC $\geq 10 \%)$ expression 20.5 vs. 8.9 months $(\mathrm{P}<0.0001)$; and for the whole population 13.8 vs. 9.6 months $(\mathrm{P}=0.0003)$. For 
subpopulation of SqCLC, the anti-PDL1 agent offered a lower OS benefit compared to standard docetaxel (8.9 vs. 7.7 months, $\mathrm{P}=0.038)$. Similarly to the other checkpoint inhibitors and in contrast to docetaxel, atezolizumab was tolerated more well with grades $3-4 \mathrm{AEs}, 15 \%$ vs. $43 \%$ (96).

\section{Durvalumab}

Durvalumab is under approval for use in NSCLC patients in the non-metastatic setting after the recently announced results of the PACIFIC trial in the last ESMO meeting 2017. In this trial, 713 patients with stage III NSCLC nonprogressing after at least two cycles of chemo-radiation were randomized to receive consolidation treatment with the human IgG1 monoclonal anti-PD-L1 antibody at $10 \mathrm{mg} / \mathrm{kg}$ or placebo, every other week, for up to a year. The PFS curves were significantly different in favor of the experimental arm, resulting in a median PFS (16.8 vs. 5.6 months, $\mathrm{P}<0.001)$. The ORR was also improved in the durvalumab arm $(28.4 \%$ vs. $16.0 \%$; $\mathrm{P}<0.001$ and $72.8 \%$ vs. $46.8 \%$ with ongoing response at 18 months, respectively). The median time to death or distant metastasis was also prolonged for approximately 10 months (23.2 vs. 14.6 months; $\mathrm{P}<0.001$ ) for the immune checkpoint inhibitor arm. In the consolidation, the safety profile of durvalumab was similar to placebo regarding toxicities greater than grade 3 (29.9\% vs. $26.1 \%$, and the most commonly observed pneumonia presented in $4.4 \%$ vs. $3.8 \%$ of cases, respectively) (82).

Durvalumab was also investigated in an immunotherapy combination with tremelimumab (anti-CTLA4 fully human IgG2 isotype inhibitor) by a phase1B trial. In this trial, durvalumab tested in 102 NSCLC patients including 10 with SqCLC. The dose for both immunotherapies was established for further trials into $10 \mathrm{mg} / \mathrm{kg}$ for durvalumab and $1 \mathrm{mg} / \mathrm{kg}$ for tremelimumab and in a 4-week cycle. Unfortunately, $36 \%$ of patients (in all testing doses) experienced serious AEs and 3 treatment-related deaths were recorded possibly due to immune-related toxicities (such as myasthenia gravis, pericardial effusion, and neuromuscular disorder). In the finally selected dose combination, the ORR was $40 \%$ and $22 \%$ for PD-L1 expression of $>25 \%$ and $0 \%$, respectively (97). With some serious concerns about its safety profile this combination is under further investigation in several studies of different treatment settings [chemo-refractory/ARCTIC study (98) and initial treatment/MYSTIC study (99)].

\section{Avelumab}

Avelumab is the less investigated of anti-PD-L1 agents in NSCLC with only some preliminary (phase $1 \mathrm{~b}$ ) results announced earlier this year. This study enrolled 184 patients with NSCLC (53 with SqCLC) to receive avelumab after failure of platinum-based chemotherapy for recurrent or metastatic NSCLC. The most common AEs of any grade observed in treatment with avelumab were fatigue $(25 \%)$, infusion-reactions $(21 \%)$ and nausea $13 \%$, while the most common AEs worse than grade 3 were infusion reactions $(2 \%)$ and increased lipase level (2\%). Bearing in mind the limitations of a phase I trial for estimating efficacy, the ORR and DCR were found $12 \%$ and $50 \%$, respectively (100).

\section{Ipilimumab}

The last introduced agent in the field of immunotherapy for NSCLC was ipilimumab, an anti-CTLA4 antibody. Based on its established role in the treatment of melanoma (101), ipilimumab is under investigation in combination with nivolumab or pembrolizumab in NSCLC $(90,91,95)$. A phase III study enrolled 956 patients with exclusively SqCLC to receive ipilimumab or placebo in addition to carboplatin-paclitaxel chemotherapy as $1^{\text {st }}$ line treatment but the immuno-chemo combination failed to improve OS. In more details, the median OS was 13.4 and 12.4 months for the immuno-chemo and conventional chemo arms respectively $(\mathrm{P}=0.25)$ whereas the two groups performed exactly the same median PFS $=5.6$ months (102).

\section{Conclusions}

The introduction of anti-VEGF, anti-EGFR, anti-PD-1, anti-PD-L1 and anti-CTLA4 agents has dramatically changed the treatment of SqCLC multiplying the therapeutic options for patients with SqCLC. Although that many advances happened during the last years in order to move from the limited treatments to the rising question about their optimal sequencing, further insights are still needed to identify new prognostic or predictive molecular biomarkers that will lead to a more individualized approach of SqCLC patients. Better histologically, molecularly and genetically determined subpopulations will increase the efficacy of targeted agents reducing in parallel the frequency of toxicity events, while the appropriate use of immunotherapy will extend the survival of treated patients improving in parallel their quality of life. 


\section{Acknowledgements}

None.

\section{Footnote}

Conflicts of Interest: The authors have no conflicts of interest to declare.

\section{References}

1. GLOBOCAN 2012: Estimated Cancer Incidence, Mortality and Prevalence Worldwide in 2012. Accessed: 30/10/2017.

2. SEER Cancer Stat Facts: Lung and Bronchus Cancer. National Cancer Institute. Bethesda, MD. Available online: http://seer.cancer.gov/statfacts/html/lungb.html

3. SEER Cancer Statistics Review, 1975-2014, National Cancer Institute. Bethesda, MD. database on the Internet. Available online: https://seer.cancer.gov/csr/1975_2014/

4. Maemondo M, Inoue A, Kobayashi K, et al. Gefitinib or chemotherapy for non-small-cell lung cancer with mutated EGFR. N Engl J Med 2010;362:2380-8.

5. Sequist LV, Yang JC, Yamamoto N, et al. Phase III study of afatinib or cisplatin plus pemetrexed in patients with metastatic lung adenocarcinoma with EGFR mutations. J Clin Oncol 2013;31:3327-34.

6. $\mathrm{Wu} \mathrm{YL}$, Zhou C, Hu CP, et al. Afatinib versus cisplatin plus gemcitabine for first-line treatment of Asian patients with advanced non-small-cell lung cancer harbouring EGFR mutations (LUX-Lung 6): an open-label, randomised phase 3 trial. Lancet Oncol 2014;15:213-22.

7. Rosell R, Carcereny E, Gervais R, et al. Erlotinib versus standard chemotherapy as first-line treatment for European patients with advanced EGFR mutation-positive non-small-cell lung cancer (EURTAC): a multicentre, open-label, randomised phase 3 trial. Lancet Oncol 2012;13:239-46.

8. Mok TS, Wu YL, Ahn MJ, et al. Osimertinib or PlatinumPemetrexed in EGFR T790M-Positive Lung Cancer. N Engl J Med 2017;376:629-40.

9. Shaw AT, Kim DW, Nakagawa K, et al. Crizotinib versus chemotherapy in advanced ALK-positive lung cancer. N Engl J Med 2013;368:2385-94.

10. Shaw AT, Kim DW, Mehra R, et al. Ceritinib in ALKrearranged non-small-cell lung cancer. N Engl J Med 2014;370:1189-97.

11. Peters S, Camidge DR, Shaw AT, et al. Alectinib versus
Crizotinib in Untreated ALK-Positive Non-Small-Cell Lung Cancer. N Engl J Med 2017;377:829-38.

12. Kim DW, Tiseo M, Ahn MJ, et al. Brigatinib in Patients With Crizotinib-Refractory Anaplastic Lymphoma KinasePositive Non-Small-Cell Lung Cancer: A Randomized, Multicenter Phase II Trial. J Clin Oncol 2017;35:2490-8.

13. Shaw AT, Ou SH, Bang YJ, et al. Crizotinib in ROS1rearranged non-small-cell lung cancer. N Engl J Med 2014;371:1963-71.

14. Planchard D, Kim TM, Mazieres J, et al. Dabrafenib in patients with BRAF(V600E)-positive advanced non-smallcell lung cancer: a single-arm, multicentre, open-label, phase 2 trial. Lancet Oncol 2016;17:642-50.

15. Novello S, Barlesi F, Califano R, et al. Metastatic nonsmall-cell lung cancer: ESMO Clinical Practice Guidelines for diagnosis, treatment and follow-up. Ann Oncol 2016;27:v1-27.

16. Schiller JH, Harrington D, Belani CP, et al. Comparison of four chemotherapy regimens for advanced non-smallcell lung cancer. N Engl J Med 2002;346:92-8.

17. NSCLC Meta-Analyses Collaborative Group. Chemotherapy in addition to supportive care improves survival in advanced non-small-cell lung cancer: a systematic review and meta-analysis of individual patient data from 16 randomized controlled trials. J Clin Oncol 2008;26:4617-25.

18. Scagliotti GV, Parikh P, von Pawel J, et al. Phase III study comparing cisplatin plus gemcitabine with cisplatin plus pemetrexed in chemotherapy-naive patients with advanced-stage non-small-cell lung cancer. J Clin Oncol 2008;26:3543-51.

19. Socinski MA, Bondarenko I, Karaseva NA, et al. Weekly nab-paclitaxel in combination with carboplatin versus solvent-based paclitaxel plus carboplatin as first-line therapy in patients with advanced non-small-cell lung cancer: final results of a phase III trial. J Clin Oncol 2012;30:2055-62.

20. Yang JJ, Huang C, Chen GY, et al. A randomized phase II clinical trial of nab-paclitaxel and carboplatin compared with gemcitabine and carboplatin as first-line therapy in locally advanced or metastatic squamous cell carcinoma of lung. BMC Cancer 2014;14:684.

21. Yoneshima Y, Morita S, Ando M, et al. Treatment Rationale and Design for J-AXEL: A Randomized Phase 3 Study Comparing Nab-Paclitaxel With Docetaxel in Patients With Previously Treated Advanced Non-SmallCell Lung Cancer. Clin Lung Cancer 2017;18:100-3.

22. Okamoto I, Yoshioka H, Morita S, et al. Phase III trial 
comparing oral S-1 plus carboplatin with paclitaxel plus carboplatin in chemotherapy-naive patients with advanced non-small-cell lung cancer: results of a west Japan oncology group study. J Clin Oncol 2010;28:5240-6.

23. Yoshioka H, Okamoto I, Morita S, et al. Efficacy and safety analysis according to histology for S-1 in combination with carboplatin as first-line chemotherapy in patients with advanced non-small-cell lung cancer: updated results of the West Japan Oncology Group LETS study. Ann Oncol 2013;24:1326-31.

24. Kubota K, Sakai H, Katakami N, et al. A randomized phase III trial of oral S-1 plus cisplatin versus docetaxel plus cisplatin in Japanese patients with advanced nonsmall-cell lung cancer: TCOG0701 CATS trial. Ann Oncol 2015;26:1401-8.

25. Shukuya T, Yamanaka T, Seto T, et al. Nedaplatin plus docetaxel versus cisplatin plus docetaxel for advanced or relapsed squamous cell carcinoma of the lung (WJOG5208L): a randomised, open-label, phase 3 trial. Lancet Oncol 2015;16:1630-8.

26. Yamada K, Saito H, Kondo T, et al. Multicenter Phase II Study of Nedaplatin and Irinotecan for Patients with Squamous Cell Carcinoma of the Lung: Thoracic Oncology Research Group 0910. Anticancer Res 2015;35:6705-11.

27. Kotsakis A, Matikas A, Koinis F, et al. A multicentre phase II trial of cabazitaxel in patients with advanced nonsmall-cell lung cancer progressing after docetaxel-based chemotherapy. Br J Cancer 2016;115:784-8.

28. Sandler A, Gray R, Perry MC, et al. Paclitaxel-carboplatin alone or with bevacizumab for non-small-cell lung cancer. N Engl J Med 2006;355:2542-50.

29. Johnson DH, Fehrenbacher L, Novotny WF, et al. Randomized phase II trial comparing bevacizumab plus carboplatin and paclitaxel with carboplatin and paclitaxel alone in previously untreated locally advanced or metastatic non-small-cell lung cancer. J Clin Oncol 2004;22:2184-91.

30. Sandler AB, Schiller JH, Gray R, et al. Retrospective evaluation of the clinical and radiographic risk factors associated with severe pulmonary hemorrhage in first-line advanced, unresectable non-small-cell lung cancer treated with Carboplatin and Paclitaxel plus bevacizumab. J Clin Oncol 2009;27:1405-12.

31. Garon EB, Ciuleanu TE, Arrieta O, et al. Ramucirumab plus docetaxel versus placebo plus docetaxel for secondline treatment of stage IV non-small-cell lung cancer after disease progression on platinum-based therapy (REVEL): a multicentre, double-blind, randomised phase 3 trial. Lancet 2014;384:665-73.

32. Bondarenko IM, Ingrosso A, Bycott P, et al. Phase II study of axitinib with doublet chemotherapy in patients with advanced squamous non-small-cell lung cancer. BMC Cancer 2015;15:339.

33. Lee Y, Shim HS, Park MS, et al. High EGFR gene copy number and skin rash as predictive markers for EGFR tyrosine kinase inhibitors in patients with advanced squamous cell lung carcinoma. Clin Cancer Res 2012;18:1760-8.

34. Zugazagoitia J, Ponce S, Paz-Ares L. Necitumumab for first-line treatment of advanced, squamous, non-small-cell lung cancer: a relevant step forward? Transl Lung Cancer Res 2016;5:95-7.

35. Thatcher N, Hirsch FR, Luft AV, et al. Necitumumab plus gemcitabine and cisplatin versus gemcitabine and cisplatin alone as first-line therapy in patients with stage IV squamous non-small-cell lung cancer (SQUIRE): an open-label, randomised, controlled phase 3 trial. Lancet Oncol 2015;16:763-74.

36. Shepherd FA, Rodrigues Pereira J, Ciuleanu T, et al. Erlotinib in previously treated non-small-cell lung cancer. N Engl J Med 2005;353:123-32.

37. Soria JC, Felip E, Cobo M, et al. Afatinib versus erlotinib as second-line treatment of patients with advanced squamous cell carcinoma of the lung (LUX-Lung 8): an open-label randomised controlled phase 3 trial. Lancet Oncol 2015;16:897-907.

38. Gatzemeier U, Pluzanska A, Szczesna A, et al. Phase III study of erlotinib in combination with cisplatin and gemcitabine in advanced non-small-cell lung cancer: the Tarceva Lung Cancer Investigation Trial. J Clin Oncol 2007;25:1545-52.

39. Herbst RS, Prager D, Hermann R, et al. TRIBUTE: a phase III trial of erlotinib hydrochloride (OSI-774) combined with carboplatin and paclitaxel chemotherapy in advanced non-small-cell lung cancer. J Clin Oncol 2005;23:5892-9.

40. Natale RB, Thongprasert S, Greco FA, et al. Phase III trial of vandetanib compared with erlotinib in patients with previously treated advanced non-small-cell lung cancer. J Clin Oncol 2011;29:1059-66.

41. Herbst RS, Ansari R, Bustin F, et al. Efficacy of bevacizumab plus erlotinib versus erlotinib alone in advanced non-small-cell lung cancer after failure of standard first-line chemotherapy (Be'Ta): a doubleblind, placebo-controlled, phase 3 trial. Lancet 
2011;377:1846-54.

42. Ciuleanu T, Stelmakh L, Cicenas S, et al. Efficacy and safety of erlotinib versus chemotherapy in second-line treatment of patients with advanced, non-small-cell lung cancer with poor prognosis (TITAN): a randomised multicentre, open-label, phase 3 study. Lancet Oncol 2012;13:300-8.

43. Scagliotti GV, Krzakowski M, Szczesna A, et al. Sunitinib plus erlotinib versus placebo plus erlotinib in patients with previously treated advanced non-small-cell lung cancer: a phase III trial. J Clin Oncol 2012;30:2070-8.

44. Garassino MC, Martelli O, Broggini M, et al. Erlotinib versus docetaxel as second-line treatment of patients with advanced non-small-cell lung cancer and wild-type EGFR tumours (TAILOR): a randomised controlled trial. Lancet Oncol 2013;14:981-8.

45. Giaccone G, Herbst RS, Manegold C, et al. Gefitinib in combination with gemcitabine and cisplatin in advanced non-small-cell lung cancer: a phase III trial--INTACT 1. J Clin Oncol 2004;22:777-84.

46. Herbst RS, Giaccone G, Schiller JH, et al. Gefitinib in combination with paclitaxel and carboplatin in advanced non-small-cell lung cancer: a phase III trial--INTACT 2.J Clin Oncol 2004;22:785-94.

47. Thatcher N, Chang A, Parikh P, et al. Gefitinib plus best supportive care in previously treated patients with refractory advanced non-small-cell lung cancer: results from a randomised, placebo-controlled, multicentre study (Iressa Survival Evaluation in Lung Cancer). Lancet 2005;366:1527-37.

48. Kim ES, Hirsh V, Mok T, et al. Gefitinib versus docetaxel in previously treated non-small-cell lung cancer (INTEREST): a randomised phase III trial. Lancet 2008;372:1809-18.

49. Pirker R, Pereira JR, Szczesna A, et al. Cetuximab plus chemotherapy in patients with advanced non-small-cell lung cancer (FLEX): an open-label randomised phase III trial. Lancet 2009;373:1525-31.

50. Lynch TJ, Patel T, Dreisbach L, et al. Cetuximab and first-line taxane/carboplatin chemotherapy in advanced non-small-cell lung cancer: results of the randomized multicenter phase III trial BMS099. J Clin Oncol 2010;28:911-7.

51. ESMO Guidelines Committee. eUpdate - Metastatic Nonsmall-cell Lung Cancer [1]: Treatment Recommendations and Revised ESMO Magnitude of Clinical Benefit Scale (ESMO-MCBS) Grading, 2017.

52. Cancer Genome Atlas Research Network. Comprehensive genomic characterization of squamous cell lung cancers. Nature 2012;489:519-25.

53. Kim HR, Kim DJ, Kang DR, et al. Fibroblast growth factor receptor 1 gene amplification is associated with poor survival and cigarette smoking dosage in patients with resected squamous cell lung cancer. J Clin Oncol 2013;31:731-7.

54. Reck M, Kaiser R, Mellemgaard A, et al. Docetaxel plus nintedanib versus docetaxel plus placebo in patients with previously treated non-small-cell lung cancer (LUMELung 1): a phase 3, double-blind, randomised controlled trial. Lancet Oncol 2014;15:143-55.

55. Lim SH, Sun JM, Choi YL, et al. Efficacy and safety of dovitinib in pretreated patients with advanced squamous non-small cell lung cancer with FGFR1 amplification: A single-arm, phase 2 study. Cancer 2016;122:3024-31.

56. Paik PK, Shen R, Berger MF, et al. A Phase Ib OpenLabel Multicenter Study of AZD4547 in Patients with Advanced Squamous Cell Lung Cancers. Clin Cancer Res 2017;23:5366-73.

57. Nogova L, Sequist LV, Perez Garcia JM, et al. Evaluation of BGJ398, a Fibroblast Growth Factor Receptor 1-3 Kinase Inhibitor, in Patients With Advanced Solid Tumors Harboring Genetic Alterations in Fibroblast Growth Factor Receptors: Results of a Global Phase I, DoseEscalation and Dose-Expansion Study. J Clin Oncol 2017;35:157-65.

58. Available online: https://clinicaltrials.gov

59. Vansteenkiste JF, Canon JL, De Braud F, et al. Safety and Efficacy of Buparlisib (BKM120) in Patients with PI3K Pathway-Activated Non-Small Cell Lung Cancer: Results from the Phase II BASALT-1 Study. J Thorac Oncol 2015;10:1319-27.

60. Levy B, Spira A, Becker D, et al. A randomized, phase 2 trial of Docetaxel with or without PX-866, an irreversible oral phosphatidylinositol 3-kinase inhibitor, in patients with relapsed or metastatic non-small-cell lung cancer. J Thorac Oncol 2014;9:1031-5.

61. Scagliotti GV, Novello S. The role of the insulinlike growth factor signaling pathway in non-small cell lung cancer and other solid tumors. Cancer Treat Rev 2012;38:292-302.

62. Langer CJ, Novello S, Park K, et al. Randomized, phase III trial of first-line figitumumab in combination with paclitaxel and carboplatin versus paclitaxel and carboplatin alone in patients with advanced non-small-cell lung cancer. J Clin Oncol 2014;32:2059-66.

63. Bergqvist M, Holgersson G, Bondarenko I, et al. Phase 
II randomized study of the IGF-1R pathway modulator AXL1717 compared to docetaxel in patients with previously treated, locally advanced or metastatic nonsmall cell lung cancer. Acta Oncol 2017;56:441-7.

64. Turner NC, Ro J, Andre F, et al. Palbociclib in HormoneReceptor-Positive Advanced Breast Cancer. N Engl J Med 2015;373:209-19.

65. Asghar U, Witkiewicz AK, Turner NC, et al. The history and future of targeting cyclin-dependent kinases in cancer therapy. Nat Rev Drug Discov 2015;14:130-46.

66. Matsushime H, Quelle DE, Shurtleff SA, et al. D-type cyclin-dependent kinase activity in mammalian cells. Mol Cell Biol 1994;14:2066-76.

67. Gopalan PK, Pinder MC, Chiappori A, et al. A phase II clinical trial of the CDK 4/6 inhibitor palbociclib (PD 0332991) in previously treated, advanced non-small cell lung cancer (NSCLC) patients with inactivated CDKN2A. J Clin Oncol 2014;32:8077.

68. Go H, Jeon YK, Park HJ, et al. High MET gene copy number leads to shorter survival in patients with non-small cell lung cancer. J Thorac Oncol 2010;5:305-13.

69. Engelman JA, Zejnullahu K, Mitsudomi T, et al. MET amplification leads to gefitinib resistance in lung cancer by activating ERBB3 signaling. Science 2007;316:1039-43.

70. Hirsch FR, Govindan R, Zvirbule Z, et al. Efficacy and Safety Results From a Phase II, Placebo-Controlled Study of Onartuzumab Plus First-Line Platinum-Doublet Chemotherapy for Advanced Squamous Cell Non-SmallCell Lung Cancer. Clin Lung Cancer 2017;18:43-9.

71. Spigel DR, Edelman MJ, O'Byrne K, et al. Results From the Phase III Randomized Trial of Onartuzumab Plus Erlotinib Versus Erlotinib in Previously Treated Stage IIIB or IV Non-Small-Cell Lung Cancer: METLung. J Clin Oncol 2017;35:412-20.

72. Neal JW, Dahlberg SE, Wakelee HA, et al. Erlotinib, cabozantinib, or erlotinib plus cabozantinib as secondline or third-line treatment of patients with EGFR wildtype advanced non-small-cell lung cancer (ECOG-ACRIN 1512): a randomised, controlled, open-label, multicentre, phase 2 trial. Lancet Oncol 2016;17:1661-71.

73. Sequist LV, von Pawel J, Garmey EG, et al. Randomized phase II study of erlotinib plus tivantinib versus erlotinib plus placebo in previously treated non-small-cell lung cancer. J Clin Oncol 2011;29:3307-15.

74. Kontopodis E, Kotsakis A, Kentepozidis N, et al. A phase II, open-label trial of bortezomib (VELCADE $((\mathrm{R})))$ in combination with gemcitabine and cisplatin in patients with locally advanced or metastatic non-small cell lung cancer. Cancer Chemother Pharmacol 2016;77:949-56.

75. Pujade-Lauraine E, Ledermann JA, Selle F, et al. Olaparib tablets as maintenance therapy in patients with platinumsensitive, relapsed ovarian cancer and a BRCA1/2 mutation (SOLO2/ENGOT-Ov21): a double-blind, randomised, placebo-controlled, phase 3 trial. Lancet Oncol 2017;18:1274-84.

76. Coleman RL, Oza AM, Lorusso D, et al. Rucaparib maintenance treatment for recurrent ovarian carcinoma after response to platinum therapy (ARIEL3): a randomised, double-blind, placebo-controlled, phase 3 trial. Lancet 2017;390:1949-61.

77. Ramalingam SS, Blais N, Mazieres J, et al. Randomized, Placebo-Controlled, Phase II Study of Veliparib in Combination with Carboplatin and Paclitaxel for Advanced/Metastatic Non-Small Cell Lung Cancer. Clin Cancer Res 2017;23:1937-44.

78. Hanahan D, Weinberg RA. Hallmarks of cancer: the next generation. Cell 2011;144:646-74.

79. Karlsson AK, Saleh SN. Checkpoint inhibitors for malignant melanoma: a systematic review and metaanalysis. Clin Cosmet Investig Dermatol 2017;10:325-39.

80. Giroux Leprieur E, Dumenil C, Julie C, et al. Immunotherapy revolutionises non-small-cell lung cancer therapy: Results, perspectives and new challenges. Eur J Cancer 2017;78:16-23.

81. Le DT, Durham JN, Smith KN, et al. Mismatch repair deficiency predicts response of solid tumors to PD-1 blockade. Science 2017;357:409-13.

82. Antonia SJ, Villegas A, Daniel D, et al. Durvalumab after Chemoradiotherapy in Stage III Non-Small-Cell Lung Cancer. N Engl J Med 2017;377:1919-29.

83. Weber J, Mandala M, Del Vecchio M, et al. Adjuvant Nivolumab versus Ipilimumab in Resected Stage III or IV Melanoma. N Engl J Med 2017;377:1824-35.

84. Gettinger SN, Horn L, Gandhi L, et al. Overall Survival and Long-Term Safety of Nivolumab (Anti-Programmed Death 1 Antibody, BMS-936558, ONO-4538) in Patients With Previously Treated Advanced Non-Small-Cell Lung Cancer. J Clin Oncol 2015;33:2004-12.

85. Brahmer J, Reckamp KL, Baas P, et al. Nivolumab versus Docetaxel in Advanced Squamous-Cell Non-Small-Cell Lung Cancer. N Engl J Med 2015;373:123-35.

86. Borghaei H, Brahmer JR, Horn L, et al. Nivolumab (nivo) vs docetaxel (doc) in patients (pts) with advanced NSCLC: CheckMate 017/057 2-y update and exploratory cytokine profile analyses. Journal of Clinical Oncology 2016;34:9025. 
87. Rizvi NA, Mazieres J, Planchard D, et al. Activity and safety of nivolumab, an anti-PD-1 immune checkpoint inhibitor, for patients with advanced, refractory squamous non-small-cell lung cancer (CheckMate 063): a phase 2, single-arm trial. Lancet Oncol 2015;16:257-65.

88. Antonia SJ, Gettinger SN, Laura QM, et al. Nivolumab (anti-PD-1; BMS-936558, ONO-4538) and ipilimumab in first-line NSCLC: Interim phase I results. J Clin Oncol 2014;32:abstr 8023.

89. Hellmann MD, Rizvi NA, Goldman JW, et al. Nivolumab plus ipilimumab as first-line treatment for advanced nonsmall-cell lung cancer (CheckMate 012): results of an open-label, phase 1, multicohort study. Lancet Oncol 2017;18:31-41.

90. Paz-Ares L, Brahmer J, Hellmann MD, et al. CheckMate 227: A randomized, open label phase 3 trial of nivolumab, nivolumab plus ipilimumab, or nivolumab plus chemotherapy versus chemotherapy in chemotherapy naïve patients with advanced non small cell lung cancer NSCLC. Annals of Oncology 2017;28:ii28-51.

91. Rizvi NA, Hellmann MD, Brahmer JR, et al.

Nivolumab in Combination With Platinum-Based Doublet Chemotherapy for First-Line Treatment of Advanced Non-Small-Cell Lung Cancer. J Clin Oncol 2016;34:2969-79.

92. Reck M, Rodriguez-Abreu D, Robinson AG, et al. Pembrolizumab versus Chemotherapy for PD-L1Positive Non-Small-Cell Lung Cancer. N Engl J Med 2016;375:1823-33.

93. Herbst RS, Baas P, Kim DW, et al. Pembrolizumab versus docetaxel for previously treated, PD-L1-positive, advanced non-small-cell lung cancer (KEYNOTE-010): a randomised controlled trial. Lancet 2016;387:1540-50.

94. Langer CJ, Gadgeel SM, Borghaei H, et al. Carboplatin and pemetrexed with or without pembrolizumab for advanced, non-squamous non-small-cell lung cancer: a randomised, phase 2 cohort of the open-label KEYNOTE-021 study. Lancet Oncol 2016;17:1497-508.

Cite this article as: Tsironis G, Ziogas DC, Kyriazoglou A, Lykka M, Koutsoukos K, Bamias A, Dimopoulos MA. Breakthroughs in the treatment of advanced squamous-cell NSCLC: not the neglected sibling anymore? Ann Transl Med 2018;6(8):143. doi: 10.21037/atm.2018.02.18
95. Fehrenbacher L, Spira A, Ballinger M, et al. Atezolizumab versus docetaxel for patients with previously treated non-small-cell lung cancer (POPLAR): a multicentre, open-label, phase 2 randomised controlled trial. Lancet 2016;387:1837-46.

96. Rittmeyer A, Barlesi F, Waterkamp D, et al. Atezolizumab versus docetaxel in patients with previously treated non-small-cell lung cancer (OAK): a phase 3, openlabel, multicentre randomised controlled trial. Lancet 2017;389:255-65.

97. Antonia S, Goldberg SB, Balmanoukian A, et al. Safety and antitumour activity of durvalumab plus tremelimumab in non-small cell lung cancer: a multicentre, phase $1 \mathrm{~b}$ study. Lancet Oncol 2016;17:299-308.

98. Planchard D, Yokoi T, McCleod MJ, et al. A Phase III Study of Durvalumab (MEDI4736) With or Without Tremelimumab for Previously Treated Patients With Advanced NSCLC: Rationale and Protocol Design of the ARCTIC Study. Clin Lung Cancer 2016;17:232-6.e1.

99. Peters S, Antonia S, Goldberg SB, et al. 191TiP: MYSTIC: a global, phase 3 study of durvalumab (MEDI4736) plus tremelimumab combination therapy or durvalumab monotherapy versus platinum-based chemotherapy (CT) in the first-line treatment of patients (pts) with advanced stage IV NSCLC. J Thorac Oncol 2016;11:S139-40.

100. Gulley JL, Rajan A, Spigel DR, et al. Avelumab for patients with previously treated metastatic or recurrent non-smallcell lung cancer (JAVELIN Solid Tumor): dose-expansion cohort of a multicentre, open-label, phase $1 \mathrm{~b}$ trial. Lancet Oncol 2017;18:599-610.

101.Hodi FS, O'Day SJ, McDermott DF, et al. Improved survival with ipilimumab in patients with metastatic melanoma. N Engl J Med 2010;363:711-23.

102. Govindan R, Szczesna A, Ahn MJ, et al. Phase III Trial of Ipilimumab Combined With Paclitaxel and Carboplatin in Advanced Squamous Non-Small-Cell Lung Cancer. J Clin Oncol 2017;35:3449-57. 